Case Report

\title{
Primary Carcinosarcoma of the Spleen: A Rare Case Report of Incidental Finding after Splenic Trauma
}

\author{
Hugues Ndasu Matendo $\stackrel{1}{1,2}$ Raouf Fayisall Geraldo ${ }^{1},{ }^{1,3}$ Liviu Musteata, ${ }^{1}$ \\ Joel Allan Green, ${ }^{4}$ Valeriu Krasovski, ${ }^{1}$ Valentin Nitu, ${ }^{1}$ Thierry Gastaud, ${ }^{1}$ and Fabrice Cattan ${ }^{1}$ \\ ${ }^{1}$ Visceral Surgery Department, Moulins-Yzeure Hospital Centre (France), 10 Avenue du Général de Gaulle, 03006 Moulins, France \\ ${ }^{2}$ Clinique la Fraternité (Congo Kinshasa), 22 Avenue Uvira, Quartier Rombe I, Uvira, Congo \\ ${ }^{3}$ Health Sciences Faculty, University of Lome, Togo \\ ${ }^{4}$ West Michigan Surgical Specialists, 1045 Gezon Parkway SW, Wyoming, MI 49509, USA
}

Correspondence should be addressed to Hugues Ndasu Matendo; ndasum@gmail.com

Received 23 June 2020; Revised 18 September 2020; Accepted 1 October 2020; Published 10 October 2020

Academic Editor: Beth A. Schrope

Copyright $\odot 2020$ Hugues Ndasu Matendo et al. This is an open access article distributed under the Creative Commons Attribution License, which permits unrestricted use, distribution, and reproduction in any medium, provided the original work is properly cited.

\begin{abstract}
Primary carcinosarcoma of the spleen is a rare, aggressive splenic malignancy. To date, seven cases have been reported in the literature. We report a first case of primary carcinosarcoma of the spleen in France. A 75-year-old woman with a medical history of hysterectomy for uterine adenocarcinoma presented with left hypochondrium pain following blunt abdominal trauma. A splenic mass was noted on computed tomography (CT) scan. A splenectomy was performed by laparotomy. Histology revealed a malignant mixed Mullerian tumor. The PET scan allowed us to confirm that it was a primary lesion of the spleen. She is currently undergoing adjuvant chemotherapy despite the tumor progression. The interest of this case lies in the rarity of primary carcinosarcomas of the spleen and the circumstances of its diagnosis.
\end{abstract}

\section{Introduction}

Carcinosarcomas are tumors composed of intimately mixed malignant epithelial and mesenchymal elements [1]. Most of carcinosarcoma appears in the genital tissue, and extragenital localizations are extremely rare [2]. They can develop at the expense of other organs, such as the peritoneum, salivary glands, lungs, esophagus, stomach, colon, pancreas, hepatobiliary system, thyroid, thymus, breast, urinary tract, and skin.

In the spleen, malignant tumors are most often of lymphoreticular origin $[1,3]$. The discovery of carcinosarcoma in the spleen is extremely rare. To the best of our knowledge, there have been seven reported cases of primary spleen carcinosarcomas in the world literature (Table 1).

Westra et al. reported the first case of primary splenic carcinosarcoma, which is said to originate from the mesothelium, reflecting the unique capacity of the female mesothelium for Mullerian differentiation $[4,5]$. Despite the rarity of this location, two male cases were presented by Rao et al.
[6] and Kochar et al. [1] in England in the same health facility. These two cases share the same characteristics, namely, age, main symptomatology, and measurements on gross examination of the spleen. However, for the case of Rao et al., there is an absence of pathological history with an increase in the levels of human chorionic gonadotropin $\beta$ $(\beta$-hCG). Joy et al. reported another case of primary splenic carcinosarcoma. However, no evidence of imaging, histology, or immunohistochemistry has been provided $[5,7]$. Sun et al. [5] reported a case of splenic carcinosarcoma with the presence of local invasion of the chest wall. Ramson et al. [8] documented a first case which benefited from a laparoscopic splenectomy. More recently, Kwok [9] presented a case which underwent a splenectomy for primary carcinosarcoma of the spleen and in which an extragenital pelvic metastasis of the said carcinosarcoma was discovered during a second laparotomy.

Here, we report a case of primary carcinosarcoma of the spleen. The objectives are to describe the circumstances of 
TABLE 1: Cases of primary carcinosarcoma of the spleen reported in the literature.

\begin{tabular}{lccccccc}
\hline Case & Author (year) (ref) & Country & Age (years) & Sex & Weight (g) (spleen) & Distant metastasis & Outcome \\
\hline 1 & Westra et al. (1994) [4] & United States & 57 & F & 1680 & FU & DD \\
2 & Rao et al. (2007) [6] & England & 60 & M & 2330 & DD \\
3 & Kochar et al. (2009) [1] & England & 60 & M & 2330 & FU & DD \\
4 & Joy et al. (2012) [7] & England & 74 & F & NP & AWM \\
5 & Sun et al. (2017) [5] & China & 64 & F & 2980 & IP & AWM \\
6 & Ramson et al. (2019) [8] & Australia & 77 & M & 895 & FU & AWM \\
7 & Kwok (2019) [9] & Australia & 74 & F & NP & DD \\
8 & Present case (2020) & France & 75 & F & 460 & No & AWM \\
\hline
\end{tabular}

IP: metastases detected at the initial presentation; FU: metastases detected during follow-up; D: died of disease; AWM: alive with the disease (at the time of publication); NP: not specified.

the diagnosis and the care provided and to review the literature.

\section{Case Presentation}

A 75-year-old female patient was admitted for abdominal pain localized in the left hypochondrium. She had a history of blunt abdominal trauma 2 months earlier and a dry cough, which had developed for several weeks without any specific care. Physical examination revealed good general condition with overweight (BMI $26.93 \mathrm{~kg} / \mathrm{m}^{2}$ ), a stable hemodynamic state, and a tenderness in the left hypochondrium but no palpable masses and lymph nodes.

Six years ago, she had undergone laparotomy for hysterectomy with bilateral oophorectomy with ilio-obturator dissection. The pathology revealed an endometrial adenocarcinoma T1a grade 1 , with the recommendation of simple follow-up every 6 months for 2 years.

The patient also has past medical history of high blood pressure, herniated disc surgery, and atopy, including allergies to iodine, and intolerance to tramadol, codeine, and nefopam.

Blood analysis showed a slight anemia with hemoglobin at $11.2 \mathrm{~g} / \mathrm{dL}$ and an elevation of platelets, $\mathrm{LDH}$ to $747 \mu / \mathrm{L}$, and C-reactive protein raised to $155 \mathrm{mg} / \mathrm{L}$. Tumor markers, including carcinoembryonic antigen (CEA), CA 19-9, and alpha-fetoprotein (AFP), as well as other hematological and biochemical parameters were normal.

During her hospitalization, a thoracoabdominal CT (Figures 1(a) and 1(b)) without injection due to allergy to iodine was performed with evidence of thoracic bilateral pleural effusion and a suspected large hematoma under the spleen capsule of $145 \times 126 \mathrm{~mm}$ with little free fluid in the abdominal cavity.

Due to the persistence of pain despite analgesic treatment (paracetamol and morphine) and uncertainty of diagnosis, we performed an exploratory laparotomy. This was done four days after admission through a midline laparotomy. A large spleen deformed by a probable large hematoma with extremely dense adhesions between this spleen and the prerenal fat as well as the diaphragm was found. There was no invasion of neighboring organs or locoregional lymphadenopathy; the other abdominal organs were normal in appearance. A splenectomy was performed.
The postoperative follow-up was simple with the prescription of Sandostatin, due to suspected pancreatic tail injury.

The patient was discharged on the seventh day after surgery with a low molecular weight heparin prescription for 3 weeks, as well as vaccines against Streptococcus pneumoniae and Neisseria meningitidis bacteria, Haemophilus influenzae type $B$, and influenza virus. She also received 2 million units of Oracillin daily for a period of 2 years.

Macroscopically, the spleen weighed $460 \mathrm{~g}$ and measured $20 \times 14 \times 8 \mathrm{~cm}$. There was a capsule breach of $8 \mathrm{~cm}$. On cutting, a voluminous nodular lesion, $12 \mathrm{~cm}$ in diameter, with a completely necrotic center was observed. At the periphery of this necrosis, there was a suspicious whitish tissue.

Histopathological examination revealed that the nodular lesion responsible for splenic rupture was a necrotic malignant neoplasm. In the tumor tissue studied, there were two distinct contingents: a tubulopapillary proliferation whose very basophilic coating included marked nuclear atypia and mitosis. These tumor glands were surrounded by atypical support tissue, made up of cells sometimes spindle shaped, sometimes large polyhedral with nuclear monstrosity.

Several immunohistochemical stains were performed: the epithelial components stained positively for cytokeratin 7 (CK7) and pancytokeratin AE1/AE3 while the sarcomatoid components stained positively for vimentin and CD10. The tumor was also positive for PAX8, WT1, and P16 and between 15 to $20 \%$ for KI67 but negative for p53, CD45, actin, desmin, and myogenin. The overall morphology and specific staining characteristics have made it possible to discuss the diagnosis of carcinosarcoma of Mullerian origin possibly secondary with suspicion of a primary tumor, either peritoneal or genital, given the history.

The PET scan, done postoperatively, showed FDG-avid areas around the splenectomy area, particularly at the level of the greater curvature of the stomach and the colonic region. This result led to a diagnosis of primary carcinosarcoma of the spleen with peritoneal involvement given the absence of other FDG-avid foci other than those around the splenectomy area.

Postoperatively, she was put on chemotherapy. Six months after surgery, because of the tumor progression, namely, a local recurrence with peritoneal carcinomatosis confirmed by CT and PET scan, after three cycles of Taxol 


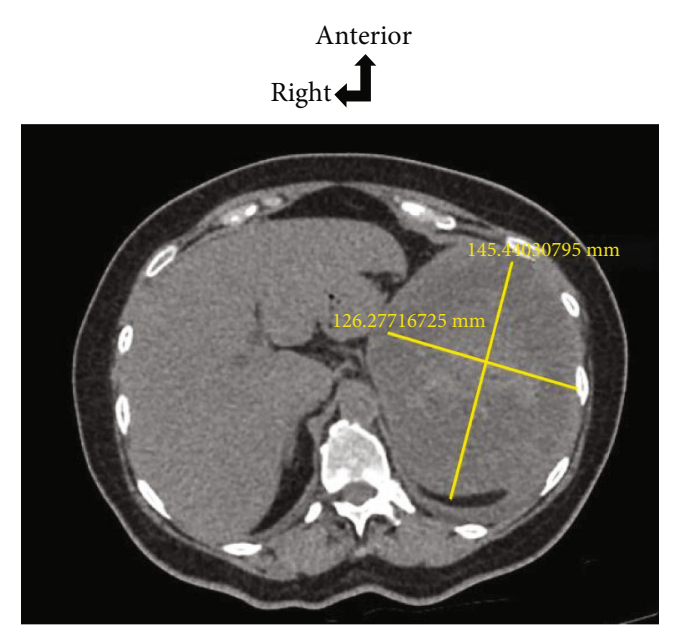

(a)

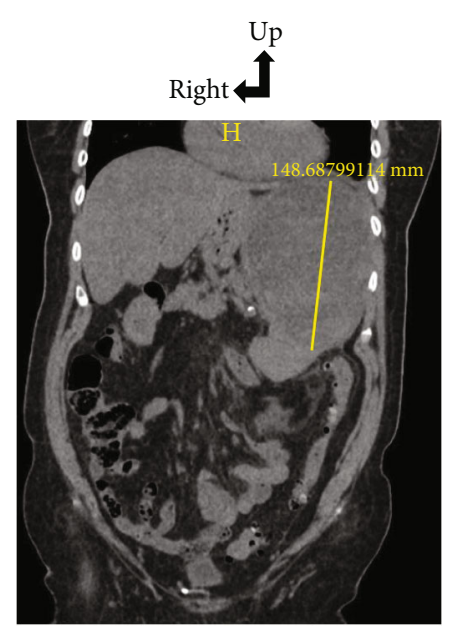

(b)

Figure 1: (a, b) Voluminous subcapsular hematoma of the upper pole of the spleen of $145 \times 126 \times 148 \mathrm{~mm}$ with low abundance intraperitoneal effusion ((a) axial section; (b) frontal reconstruction).

carboplatin then two cycles of anthracycline (doxorubicin), the patient was put on Gemcitabine.

To date, 8 months after surgery, she is in her second cycle of Gemcitabine. In addition to the peritoneal carcinomatosis which slightly declined under this latter treatment, the patient did not present any other secondary lesions.

\section{Discussion}

Virchow, in 1864, was the first to use the term carcinosarcoma for the description of a tumor associating a carcinomatous component with a sarcomatous component [10]. These are neoplasms whose typical primary site is the female genital tract, with high metastatic potential [11]. The diagnosis is most often incidental. Both primary and secondary involvement of the spleen is rare [3]. To date, seven cases of primary carcinosarcomas of the spleen have been reported in the literature (Table 1).

Patient age ranged between 57 and 77 years, including four females who were postmenopausal.

The clinical presentation is not specific: in seven cases, five $[1,4-6,9]$ had a painful mass in the left upper quadrant while the other two had anemia and painless mass of the upper left quadrant. In our case, it is a 75-year-old woman who was asymptomatic before the abdominal trauma. She presented with pain in the left hypochondrium after this trauma.

Histologically, our patient's spleen was the smallest in the series and weighed $460 \mathrm{~g}$.

The carcinomatous component often combines a highgrade serous component, grade 3 endometrioide, with clear or undifferentiated cells. Depending on the sarcomatous component, two types are defined: either the sarcomatous component is normally present within the organ, we will then speak of homologous carcinosarcoma, or the component is formed from elements usually absent (cartilage tissue, bone, muscle fibers striated,...), we will then speak of heterologous carcinosarcoma (the most frequent form) [12].
The carcinogenesis and the biological behavior of carcinosarcoma are not fully understood.

Four theories have been put forward after comparing the epidemiological, clinical, histopathological, biological, and immunohistochemical profiles $[5,13,14]$. First, the collision theory, underpinned by a biclonal origin: two tumors (epithelial and mesenchymal) would emerge simultaneously from two separate stem cells. Second is the composition theory, which suggests that the mesenchymal tumor represents a pseudosarcomatous reaction of the epithelial tumor. Third, the combination theory, underpinned by a monoclonal origin: a common stem cell would alternately generate epithelial and mesenchymal cancer cells. Fourth, the theory of conversion, which also supports a monoclonal origin: from a stem cell would develop a tumor either epithelial or mesenchymal which would secondarily differentiate into the other tumor type, in particular an epithelial origin with a mesenchymal metaplasia.

Despite the remaining uncertainty about the mechanisms that generate these tumors, recent immunohistochemical, ultrastructural, and molecular genetic studies suggest and promote the concept of monoclonality in carcinosarcoma [13]. In addition, identical p53 and KRAS mutations have been identified in the epithelial and mesenchymal components of carcinosarcoma, results which suggest an early alteration in the histogenesis of the tumor with late transformation or degeneration of the epithelial component into the sarcomatous component [13].

However, the immunohistochemical study has little interest apart from the demonstration of a heterologous contingent; in other words, it is not essential if the histological aspect is characteristic. The epithelial components are generally positive for cytokeratin, while the sarcomatous tissue is generally positive for vimentin $[4,9]$, which is consistent with the staining pattern in our case.

To date, there is no consensus for the treatment of splenic carcinosarcoma. It is generally accepted that early surgery with complete macroscopic resection (splenectomy) 
of the malignant tumor may offer the best chance of survival $[1,5,9]$.

However, one of the cases benefited from two surgeries for the same disease [9]: first, a total subcostal splenectomy; this did not allow the pelvis to be explored at the same time, with immediate postoperative discovery of a pelvic peritoneal metastasis that required a second laparotomy. One should consider the approaches that allow good exploration and management of a splenic mass:

(i) Laparoscopy, if possible, or robotic surgery with [8] or without a mini laparotomy to extract the spleen

(ii) Exploratory laparotomy by the subcostal approach in the absence of other pathology

(iii) Splenectomy by a midline incision

Postoperatively, adjuvant treatments, such as chemotherapy and radiotherapy, could be considered to improve the prognosis of patients [5]. For chemotherapy, both carcinomatous and sarcomatous components must be treated. Numerous chemotherapy regimens including ifosfamide, paclitaxel, cisplatin, and carboplatin have been tested, with no solid evidence indicating the superiority of one combination over another. However, it has been shown that the use of chemotherapy as single therapy results in lower overall survival [9, 15]. Postoperative irradiation has been shown to improve local control without benefit to overall survival. Multimodal therapy should lead to better results. Recently, there are many ongoing studies with molecular and targeted therapies to improve efficacy; however, relevant results may not be available for several years due to the small number of patients [11].

The prognosis for carcinosarcoma is poor, with splenic localization being no exception [8]. Postoperative survival is estimated between 3 and 12 months [1, 4-9].

\section{Conclusion}

Primary carcinosarcoma of the spleen is a rare entity with a poor prognosis. Very few cases have been reported in the literature. Two histological types are described: the heterologous type and the homologous type, but without affecting the prognosis. There is no consensus for adjuvant therapy with chemotherapy, radiotherapy, or targeted therapy. The surgical management should include splenectomy with complete exploration of the abdominal cavity.

\section{Abbreviations}

AE1/AE3: Cytokeratin (or pancytokeratin) AE1/AE3: keratin cocktail that detects CK1-8, 10, 14-16, and 19, but does not detect CK17 or CK18

CA: $\quad$ Cancer antigen

CD10: $\quad$ Cluster of differentiation 10

CD45: $\quad$ Cluster of differentiation 45

CEA: Carcinoembryonic antigen

CK7: $\quad$ Cytokeratin 7

KI67: A cellular marker for proliferation
KRAS: $\quad$ Kirsten rat sarcoma viral oncogene homolog

LDH: $\quad$ Lactate dehydrogenase

P16: $\quad$ (Also known as p16INK4a, cyclin-dependent kinase inhibitor 2A, CDKN2A, multiple tumor suppressor 1) tumor suppressor protein

p53: $\quad$ Tumor protein 53

PAX8: $\quad$ Paired box gene 8 (for primary or secondary Mullerian tumor)

PET: $\quad$ Positron emission tomography

WT1: Wilms tumor 1.

\section{Conflicts of Interest}

The authors declare that there are no conflicts of interest regarding the publication of this paper.

\section{Authors' Contributions}

All authors actively participated in the realization of this work. NMH was responsible for subject design and configuration, manuscript correction, translation of the manuscript into English, and supervision of the work. GFR, CF, and ML were responsible for manuscript correction and supervision of the work. GAJ was responsible for correction of the English manuscript. NMH, GFR, ML, GAJ, GT, NV, KV, and CF were responsible for the review of the final manuscript.

\section{References}

[1] K. Kochar, C. Vijayasekar, U. Pandey, R. Bhogal, L. Brown, and G. Mathew, "Primary carcinosarcoma of the spleen: case report of a rare tumor and review of the literature," International Journal of Surgical Pathology, vol. 17, no. 1, pp. 72-77, 2008.

[2] G. Paulsson, S. Andersson, and B. Sorbe, "A population-based series of ovarian carcinosarcomas with long-term follow-up," Anticancer Research, vol. 33, no. 3, pp. 1003-1008, 2013.

[3] R. K. Kaza, S. Azar, M. M. Al-Hawary, and I. R. Francis, "Primary and secondary neoplasms of the spleen," Cancer Imaging, vol. 10, pp. 173-182, 2010.

[4] W. H. Westra, B. O. Anderson, and D. S. Klimstra, "Carcinosarcoma of the spleen. An extragenital malignant mixed müllerian tumor?," The American Journal of Surgical Pathology, vol. 18, no. 3, pp. 309-315, 1994.

[5] T. Sun, G.-F. Wang, and Y. Zhang, "Primary splenic carcinosarcoma with local invasion of chest wall: a rare case," Journal of Zhejiang University. Science. B, vol. 18, no. 8, pp. 717-722, 2017.

[6] A. Rao, G. Mathew, K. Marimuthu, U. Pandey, and J. Worlding, "Carcinosarcoma of the spleen: literature review of a very rare tumour," Open Medicine, vol. 2, no. 2, pp. 230235, 2007.

[7] E. Joy, J. Cornish, R. Bootun, S. Datta, and P. Chandran, "Primary carcinosarcoma of the spleen," Journal of Surgical Case Reports, vol. 2012, no. 7, p. 3, 2012.

[8] D. M. Ramson, T. Ackermann, M. Thompson, and J. Gribbin, "Primary pleomorphic carcinosarcoma of the spleen: case report and review of the literature," ANZ Journal of Surgery, vol. 90, no. 6, pp. 1209-1211, 2020. 
[9] A. M. F. Kwok, "Primary extragenital mixed malignant Mullerian tumour presenting as a painful splenic mass: a case report and review of the literature," World Journal of Obstetrics and Gynecology, vol. 8, no. 2, pp. 13-24, 2019.

[10] M. Muller, P.-F. Dupre, B. Lucas et al., "Le carcinosarcome ovarien," Journal de Gynécologie Obstétrique et Biologie de la Reproduction, vol. 36, no. 4, pp. 399-402, 2007.

[11] J. Kalincsák, P. Göcze, J. Bódis et al., "Multimodal treatment of malignant mixed Müllerian tumor," Orvosi Hetilap, vol. 159, no. 19, pp. 741-747, 2018.

[12] A.-C. Hellstrom, G. Tegerstedt, C. Silfversward, and F. Pettersson, "Malignant mixed mullerian tumors of the ovary: histopathologic and clinical review of 36 cases," International Journal of Gynecological Cancer, vol. 9, no. 4, pp. 312-316, 1999.

[13] T. L. Loh, J. Tomlinson, R. Chin, and G. D. Eslick, "Cutaneous carcinosarcoma with metastasis to the parotid gland," Case Reports in Otolaryngology, vol. 2014, Article ID 173235, 4 pages, 2014.

[14] F. Amant, V. Vloeberghs, H. Woestenborghs, P. Moerman, and I. Vergote, "Transition of epithelial toward mesenchymal differentiation during ovarian carcinosarcoma tumorigenesis," Gynecologic Oncology, vol. 90, no. 2, pp. 372-377, 2003.

[15] R. Kanthan and J.-L. Senger, "Uterine carcinosarcomas (malignant mixed müllerian tumours): a review with special emphasis on the controversies in management," Obstetrics and Gynecology International, vol. 2011, Article ID 470795, 13 pages, 2011. 